\title{
Autores prolíficos líderes en la investigación turística española
}

\author{
Leader prolific authors in Spanish tourism research
}

\author{
Jesús Manuel LÓPEZ-BONILLA ${ }^{1}$ \\ Concepción GRANADOS-PEREA ${ }^{1}$ \\ Luis Miguel LÓPEZ-BONILLA ${ }^{1}$
}

\begin{abstract}
Resumen
El sector turístico es muy importante en el mundo. En concreto, en España es uno de los sectores económicos principales. Por tanto, la investigación turística española debe desempeñar un papel destacado. Este trabajo se ha centrado en el análisis de la producción internacional de los investigadores españoles en el ámbito del turismo. Este estudio bibliométrico se ha basado en el uso de la base de datos Scopus desde 2002 hasta 2013. Así, un primer objetivo ha sido la identificación de los investigadores españoles principales en turismo. Un segundo objetivo fue identificar a los autores prolíficos que se pueden considerar líderes en la investigación turística en España. Este segundo objetivo ha tratado de proporcionar un procedimiento bibliométrico para seleccionar a los autores líderes, de manera que se pueda establecer una distinción entre autores que publican sus investigaciones sobre turismo. Se ha obtenido un ranking de 79 autores prolíicos líderes en la investigación turística mediante una metodología basada especialmente en el orden de firmas.
\end{abstract}

Palabras Clave: Autoría. Bibliometría. Investigación turística. Investigadores. Orden de firma.

\begin{abstract}
Tourism and hospitality is a very important industry in the world. Specially, it is one of the most important sectors in Spain. Therefore, Spanish tourism research must play a relevant role. This paper focuses on the analysis of the international scientific output of the Spanish researchers on tourism and hospitality. The bibliometric analysis was performed using the database Scopus from 2002 to 2013. Hence, the article's first objective was the identification of the main Spanish researchers in tourism and hospitality. The second objective was to identify the leader and most prolific authors in tourism and hospitality research in Spain. This second objective sought to provide a procedure for selecting leader authors, therefore enabling a distinction among different authors who publish their research in tourism and hospitality. A ranking of 79 prolific leader authors in tourism and hospitality research was obtained using a methodology based especially on authorship order.
\end{abstract}

Keywords: Authorship. Bibliometrics. Tourism and hospitality research. Research workers. Signature order.

\section{Introducción}

El sector turístico representa una industria muy importante en todo el mundo. En concreto, España lleva muchos años situándose entre los países principales del sector. Actualmente, España es el tercer país del mundo con mayor número de turistas internacionales que lo visitan y también es el tercer país en el que se obtienen mayores ingresos por este concepto (Organización Mundial del Turismo, 2016). Sin embargo, la investigación turística en España ha estado mucho más rezagada hasta hace relativamente poco tiempo.

\footnotetext{
1 Universidad de Sevilla, Facultad de Ciencias Económicas y Empresariales, Departamento de Administración de Empresas y Marketing. Av. Ramón y Cajal, 1, 41018, Sevilla, España. Correspondencia a nombre de/Correspondence to: J.M. López-Bonilla. E-mail:<lopezbon@us.es>.

Recibido el 11 del abril del 2017, versión final re-presentada el 23 del junio del 2017 y aprobado el 17 del julio del 2017.

Como citar este artigo/How to cite this article

López-Bonilla, J. M.; Granados-Perea, C.; López-Bonilla, L. M. Autores prolíficos líderes en la investigación turística española. Transinformação, v. 30, n. 1, p. 39-50, 2018. https://doi.org/10.1590/2318-08892018000100004
} 
El presente trabajo se va a enfocar hacia el análisis de autoría de los investigadores españoles en turismo. En España son muy escasos los artículos que se han dedicado exclusivamente al análisis de los autores prolíficos en la investigación turística española. En el ámbito internacional, como sugieren recientemente López-Bonilla, Granados-Perea y López-Bonilla (2017), los estudios bibliométricos centrados en las autorías tampoco han tenido un gran desarrollo hasta bien entrado este siglo XXI. Así, por ejemplo, como ya indicaron Zhao y Ritchie (2007), el análisis de autoría ha sido una práctica común en otras disciplinas maduras, mientras que es un fenómeno mucho más reciente en la investigación turística. Además, en un contexto internacional, se han encontrado varios estudios basados en un análisis de autorías de la investigación turística (McKercher, 2007, 2008; Zhao; Ritchie, 2007; Zhong; Wu; Morrison, 2015), pero todos ellos se han centrado en la identificación de los autores prolíicos. Sin embargo, ninguno de estos trabajos se ha orientado hacia la posible distinción de los investigadores prolíficos líderes respecto a otros que adoptan una participación más colaboradora del autor líder.

En este sentido, hay que preguntarse por lo que vamos a entender como autores prolíficos líderes en el presente trabajo. Se puede decir que son aquellos que publican trabajos en revistas científicas de prestigio internacional, pero que también actúan como líderes o autores principales de sus propios trabajos e investigaciones. En el ámbito de las publicaciones científicas es conveniente distinguir los conceptos de líder académico y autor líder. El líder académico puede publicar trabajos propios y trabajos ajenos, sobre todo teniendo en cuenta que va a apoyar a otros autores más noveles en su desarrollo académico. Éste sería el caso más común que ocurre en la relación entre director de tesis y doctorando. En cambio, el autor prolífico líder debería destacar especialmente por publicar una proporción elevada de trabajos propios respecto al total de publicaciones que ha firmado. Además, los autores o investigadores considerados líderes no tienen porqué coincidir con investigadores líderes de su área de conocimiento o de su centro de investigación. Es decir, estos autores líderes pueden ser considerados más propiamente como científicos, en el sentido estricto de estar relacionados más exclusivamente con la investigación, pero no con el liderazgo en las organizaciones en las que están situados jerárquicamente.

Las escasas clasificaciones de los autores prolíficos líderes en la investigación turística, a nivel mundial, se han basado en el número de publicaciones que han realizado cada uno de ellos. Es decir, se supone un mayor liderazgo cuanto mayor es su número de publicaciones. Sin embargo, no se ha adoptado ninguna metodología más precisa para tratar de identificar a estos autores líderes. En el presente estudio se pretende cubrir este gap de la literatura. Hoy en día, los autores se clasifican generalmente en función de la cantidad significativa de sus contribuciones a la investigación (He; Ding; Yan, 2012). Como indican Brown, Chan y Lai (2006), la literatura argumenta que el orden de autoría envía una señal sobre su contribución relativa de un artículo publicado, y esta señal se pierde cuando los nombres de autores se ordenan alfabéticamente. Además, Ruiz-Pérez, Marcos-Cartagena y Lópes-Cózar (2014) señalan que el orden de firma puede ser relevante y un tema de conflicto, sobre todo si se considera como un mérito científico, como ocurre en España, para poder alcanzar un reconocimiento científico que sirve también para la promoción de profesores e investigadores.

Por todo ello, el objetivo principal del presente trabajo se ocupa de identificar a los autores prolíficos líderes en España que han publicado sus trabajos en el ámbito turístico en revistas indexadas en bases de datos internacionales de prestigio. Se pretende detectar el perfil de los investigadores más prolíicos a través de las revistas donde han publicado, la afiliación de estos autores, las zonas geográficas, el número de autores por publicación y el género. Este trabajo puede representar una mayor contribución a la investigación académica tanto en el ámbito del estudio turístico como en el ámbito de la información científica. La posición de España en el sector turístico mundial, así como la importancia de la investigación turística española, suponen un motivo extraordinario para desarrollar este trabajo. Así, se pretende aportar un mayor conocimiento sobre este tópico a la comunidad científica nacional e internacional en el ámbito turístico. Los hallazgos pueden ser útiles para instituciones, investigadores y estudiantes de doctorado y máster. Como indica Díaz-Campos (2016), los estudiantes de doctorado españoles 
parecen tener muy en cuenta el prestigio de las instituciones y de los directores de tesis para elegir su temática de estudio.

\section{Procedimientos metodológicos}

La metodología de este estudio hay que plantearla desde distintos aspectos, como son los siguientes: (1) la identificación del concepto estudiado; (2) la selección de revistas y de trabajos publicados, (3) la selección de la búsqueda de palabras clave; (4) el período de tiempo analizado; y (5) la medida de las autorías. En primer lugar, es necesario definir el concepto que va a ser investigado en el análisis bibliométrico. Este concepto de búsqueda se corresponde con los trabajos realizados sobre la investigación turística por autores afiliados a alguna institución española, que han sido publicados en prestigiosas revistas internacionales y durante un período de 12 años que va desde 2002 a 2013.

La selección de revistas ha sido un aspecto controvertido en la literatura académica. Las revistas de prestigio se pueden definir como aquellas que están indexadas en grandes bases de datos internacionales y que dispongan de un índice de impacto reconocido por la comunidad científica internacional. Actualmente, las dos bases de datos más relevantes y más utilizadas en los estudios bibliométricos son ISI Web of Knowledge (WoS), de la empresa Thomson Reuters, y Scopus, del grupo Elsevier. Para el análisis bibliométrico de la investigación turística española se ha optado por el uso de la base de datos Scopus, debido a su mayor cobertura de revistas.

Se ha creído conveniente analizar en este trabajo todas las publicaciones incluidas en Scopus y no solo las revistas especializadas en turismo. En primer término, la selección de trabajos publicados se ha basado en una revisión exhaustiva de todas las revistas especializadas netamente en investigación turística que están incluidas en la categoría de "Tourism, Leisure and Hospitality Management" de Scopus. También se han incluido otras revistas especializadas que se consideran importantes, pero que aparecen en otras categorías distintas, tales como Journal of Travel Research y Anatolia: An International Journal of Tourism and Hospitality Research. En segundo término, se han revisado también de manera exhaustiva todas las revistas científicas más específicas sobre la investigación del ocio, depurando aquellos trabajos publicados que no se correspondían con el ámbito turístico. Y, en tercer lugar, se ha realizado una revisión del resto de trabajos publicados sobre materia turística en revistas científicas incluidas en Scopus, sin estar especializadas en el ámbito turístico y del ocio. En total han sido 704 revistas científicas de las que se ha obtenido, al menos, una publicación realizada por autores de instituciones españolas.

Para seleccionar los trabajos publicados en revistas no especializadas en turismo que están incluidas en Scopus se ha optado por la búsqueda de cuatro palabras clave que se identifiquen claramente con la investigación turística y que evite confusiones con otros ámbitos de estudio al estar bien diferenciadas. Así, se han elegido dos palabras genéricas, como son "tourism" y "tourist", que abarcan la gran mayoría de trabajos publicados, y otras dos palabras clave específicas relacionadas con las principales empresas turísticas del sector, como son "hotel" y "travel agency". Esta búsqueda se ha realizado a través de los títulos de artículos, los resúmenes y las palabras clave (title, abstracts and keywords) de Scopus. Además, se ha realizado una última búsqueda complementaria a la anterior que se ha basado en buscar un amplio conjunto de palabras clave para encontrar nuevos trabajos de los autores prolíicos ya detectados, o bien, para hallar nuevos autores prolíicos, en su caso. Para ello, en concreto, se ha utilizado la totalidad de palabras clave que se recoge en el trabajo de Corral y Cànoves (2013), que suman la cincuentena de conceptos de búsqueda.

Por otra parte, en el proceso de búsqueda se han incluido exclusivamente aquellos trabajos que aparecen como publicaciones en revistas (identificadas en Scopus con el término de "journals") y que hayan sido objeto de revisión científica, es decir, se han eliminado aquellos documentos que hayan sido considerados como editoriales, libros, capítulos de libros y reseñas de libros. 
En consonancia con otros análisis de autores en el ámbito internacional (McKercher, 2008; Park et al., 2011; Zhao; Ritchie, 2007) en los que se supera ligeramente el medio centenar de autores, se ha realizado una selección de autores más prolíficos estableciendo el punto de corte en seis trabajos publicados, coincidiendo con Albacete, Fuentes y Haro-Dominguez (2013). Así, los autores prolíficos deben haber publicado, al menos, seis trabajos en prestigiosas revistas internacionales. Por tanto, se ha recogido un total de 1864 publicaciones en revistas científicas indexadas en Scopus que cumplen con los requisitos previstos en el presente estudio durante el período 2002-2013.

Para seleccionar a los autores o investigadores líderes en turismo se ha considerado el orden de autorías de los trabajos publicados. Se puede entender que un autor principal debe ser aquel que figure como primera firma en la mayoría de los trabajos publicados en los que haya participado. Este requisito de primera autoría ha sido cada vez más observado para evaluar la carrera científica en los últimos años. Siendo así, se considera un indicador válido para identificar y clasificar a los autores o investigadores líderes. La expresión que se va a utilizar para establecer el ranking de autores en función de su liderazgo por primera autoría es la siguiente:

$$
\text { Ratio autor lider }=\frac{\text { No trabajos publicados }}{\text { Ratio primera autoría } \times \text { Ratio coautoría }}
$$

El ratio de autor líder se entiende también como el cociente entre el número de trabajos publicados fraccionados y el ratio de primera autoría, puesto que el ratio de co-autoría representa el número medio de colaboradores que han elaborado los trabajos publicados de cada autor líder. Por su parte, el ratio de primera autoría se calcula a partir de la media ponderada del número de firmantes de cada trabajo publicado. De este modo, si un supuesto autor o investigador firmara todos sus trabajos como primer autor, el valor correspondiente sería la unidad, si es el único autor en todos sus trabajos, o superior a la unidad, si hay varios autores en el conjunto de sus trabajos. En el caso más corriente de tratarse de un autor o investigador que firmara sus trabajos en distintas posiciones de autoría, habría que obtener la media ponderada de todas las posiciones que ha ocupado en las firmas de sus trabajos publicados. Así, por ejemplo, para un autor que haya publicado seis trabajos, siendo dos de ellos como primera firma (2 artículos por 1), otros dos como segunda firma (2 artículos por 2) y otros dos como tercera firma (2 artículos por 3), el valor medio de primera autoría sería un dos.

\section{Resultados}

La producción científica española sobre turismo con difusión internacional comienza su verdadero despegue con el inicio del siglo XXI. Precisamente, estas fechas son coincidentes con la entrada en vigor en España de la Ley de Universidades a finales de 2001 que, entre otros aspectos, promueve la investigación académica a través de criterios de evaluación para el acceso a cuerpos de profesores universitarios que se basan en las publicaciones en revistas indexadas en bases de datos internacionales. El crecimiento anual de publicaciones ha mantenido una tendencia ascendente, acentuándose en los últimos años. La tasa media de aumento interanual desde 1995 hasta 2014 ha sido del $25 \%$.

La Tabla 1 recoge una lista de 79 autores prolíficos adscritos a universidades españolas que tienen, al menos, seis trabajos publicados en la base de Scopus durante el doble sexenio entre 2002 y 2013 y que están relacionados con el ámbito de la investigación turística. Todos estos autores suman un total de 285 publicaciones en revistas indexadas en Scopus. Estos autores representan a 28 instituciones, siendo un centro de investigación del CSIC, una universidad privada y 27 universidades públicas. Uno de los autores aparece adscrito a dos instituciones.

En la Tabla 1 se detallan los nombres de los investigadores, su afiliación académica e institucional, el número de trabajos totales que ha publicado en el período de estudio, el ratio de primera autoría, el ratio de co-autoría y el 
Tabla 1. Ranking de autores en la investigación turística española (2002-2013).

\begin{tabular}{|c|c|c|c|c|c|c|c|}
\hline Orden general & Autores & Institución & $\begin{array}{l}\text { Trabajos } \\
\text { Totales (n) }\end{array}$ & $\begin{array}{l}\text { Primera } \\
\text { autoría }\end{array}$ & Co-autoría & Ratio líder & Orden líder \\
\hline 1 & J.L. Nicolau & $\begin{array}{l}\text { Universidad de } \\
\text { Alicante }\end{array}$ & 27 & 1,15 & 1,52 & 15,45 & 1 \\
\hline 2 & E. Claver-Cortés & $\begin{array}{l}\text { Universidad de } \\
\text { Alicante }\end{array}$ & 26 & 1,27 & 3,23 & 6,34 & 3 \\
\hline 3 & J. Rosselló & $\begin{array}{c}\text { Universidad Islas } \\
\text { Baleares }\end{array}$ & 24 & 2,08 & 2,33 & 4,95 & 5 \\
\hline 4 & I. Gil Saura & $\begin{array}{l}\text { Universidad de } \\
\text { Valencia }\end{array}$ & 21 & 1,90 & 2,71 & 4,08 & 8 \\
\hline 5 & D.M. Frías Jamilena & $\begin{array}{l}\text { Universidad de } \\
\text { Granada }\end{array}$ & 21 & 1,90 & 3,10 & 3,57 & 12 \\
\hline 6 & J. Pereira-Moliner & $\begin{array}{l}\text { Universidad de } \\
\text { Alicante }\end{array}$ & 20 & 2,35 & 3,25 & 2,62 & 20 \\
\hline 7 & J.F. Molina-Azorín & $\begin{array}{l}\text { Universidad de } \\
\text { Alicante }\end{array}$ & 20 & 1,05 & 3,45 & 5,52 & 4 \\
\hline 8 & J. Alegre & $\begin{array}{l}\text { Universidad Islas } \\
\text { Baleares }\end{array}$ & 19 & 1,11 & 2,47 & 6,93 & 2 \\
\hline 9 & M.M. Alonso-Almeida & $\begin{array}{l}\text { Univ. Autónoma de } \\
\text { Madrid }\end{array}$ & 19 & 1,79 & 2,58 & 4,11 & 7 \\
\hline 10 & M.J. Such Devesa & $\begin{array}{l}\text { Universidad de } \\
\text { Alcalá de Henares }\end{array}$ & 16 & 2,25 & 2,50 & 2,84 & 16 \\
\hline 11 & $\begin{array}{l}\text { M.A. Rodríguez- } \\
\text { Molina }\end{array}$ & $\begin{array}{l}\text { Universidad de } \\
\text { Granada }\end{array}$ & 15 & 2,87 & 3,27 & 1,60 & 44 \\
\hline 12 & J. Rey-Maquieira & $\begin{array}{c}\text { Universidad Islas } \\
\text { Baleares }\end{array}$ & 15 & 3,07 & 3,73 & 1,31 & 52 \\
\hline 13 & L.A. Gil-Alaña & $\begin{array}{l}\text { Universidad de } \\
\text { Navarra }\end{array}$ & 12 & 1,67 & 2,42 & 2,97 & 14 \\
\hline 14 & J.A. Fraiz Brea & Universidad de Vigo & 12 & 2,17 & 2,75 & 2,01 & 32 \\
\hline 15 & S. Moreno-Gil & $\begin{array}{l}\text { Univ. Las Palmas de } \\
\text { Gran Canaria }\end{array}$ & 11 & 1,91 & 2,55 & 2,26 & 27 \\
\hline 16 & M.E. Ruiz-Molina & $\begin{array}{l}\text { Universidad de } \\
\text { Valencia }\end{array}$ & 11 & 1,82 & 2,82 & 2,14 & 30 \\
\hline 17 & A. Riera-Font & $\begin{array}{c}\text { Universidad Islas } \\
\text { Baleares }\end{array}$ & 11 & 2,09 & 2,82 & 1,87 & 38 \\
\hline 18 & A.I. Polo-Peña & $\begin{array}{l}\text { Universidad de } \\
\text { Granada }\end{array}$ & 11 & 1,00 & 2,91 & 3,78 & 11 \\
\hline 19 & T. López-Guzmán & $\begin{array}{l}\text { Universidad de } \\
\text { Córdoba }\end{array}$ & 11 & 1,36 & 2,91 & 2,78 & 18 \\
\hline 20 & G. Canovés Valiente & $\begin{array}{c}\text { Univ. Autónoma de } \\
\text { Barcelona }\end{array}$ & 10 & 1,90 & 2,30 & 2,29 & 26 \\
\hline 21 & L. Parte Esteban & UNED & 10 & 1,78 & 2,40 & 2,34 & 25 \\
\hline 22 & $\begin{array}{l}\text { I. Rodríguez del } \\
\text { Bosque }\end{array}$ & $\begin{array}{l}\text { Universidad de } \\
\text { Cantabria }\end{array}$ & 10 & 2,10 & 2,40 & 1,98 & 33 \\
\hline 23 & A. Molina Collado & $\begin{array}{l}\text { Univ. de Castilla-La } \\
\text { Mancha }\end{array}$ & 10 & 2,20 & 2,80 & 1,62 & 43 \\
\hline 24 & J.J. Tari Guillo & $\begin{array}{l}\text { Universidad de } \\
\text { Alicante }\end{array}$ & 10 & 2,30 & 3,50 & 1,24 & 59 \\
\hline
\end{tabular}


Tabla 1. Ranking de autores en la investigación turística española (2002-2013).

\begin{tabular}{|c|c|c|c|c|c|c|c|}
\hline Orden general & Autores & Institución & $\begin{array}{c}\text { Trabajos } \\
\text { Totales (n) }\end{array}$ & $\begin{array}{r}\text { Primera } \\
\text { autoría }\end{array}$ & Co-autoría & Ratio líder & Orden líder \\
\hline \multirow[t]{2}{*}{25} & B. Plaza & Universidad del País & 9 & 1,00 & 1,89 & 4,76 & 6 \\
\hline & & Vasco & & & & & \\
\hline \multirow[t]{2}{*}{26} & A. Mantecón & Universidad de & 9 & 1,33 & 2,00 & 2,28 & 13 \\
\hline & & Alicante & & & & & \\
\hline 27 & J.M. López-Bonilla & Universidad de Sevilla & 9 & 1,11 & 2,11 & 3,84 & 10 \\
\hline 28 & L.M. López-Bonilla & Universidad de Sevilla & 9 & 1,89 & 2,11 & 2,26 & 28 \\
\hline \multirow[t]{2}{*}{29} & T.F. Espino-Rodríguez & Univ. Las Palmas de & 9 & 1,50 & 2,13 & 2,82 & 17 \\
\hline & & Gran Canaria & & & & & \\
\hline 30 & J.I. Pulido-Fernández & Universidad de Jaén & 9 & 1,56 & 2,33 & 2,48 & 22 \\
\hline \multirow[t]{2}{*}{31} & C.J. León González & Univ. Las Palmas de & 9 & 1,67 & 2,56 & 2,11 & 31 \\
\hline & & Gran Canaria & & & & & \\
\hline \multirow[t]{2}{*}{32} & E. Aguiló & Universidad Islas & 9 & 1,63 & 2,88 & 1,92 & 35 \\
\hline & & Baleares & & & & & \\
\hline \multirow[t]{2}{*}{33} & E. Bigné-Alcañiz & Universidad de & 9 & 1,22 & 3,00 & 2,46 & 23 \\
\hline & & Valencia & & & & & \\
\hline \multirow[t]{2}{*}{34} & J.M. Peiró & Universidad de & 9 & 3,13 & 3,22 & 0,89 & 72 \\
\hline & & Valencia & & & & & \\
\hline \multirow[t]{2}{*}{35} & J.A. Castañeda-García & Universidad de & 9 & 2,33 & 3,33 & 1,66 & 64 \\
\hline & & Granada & & & & & \\
\hline 36 & J. Sánchez-García & Universidad Jaume I & 9 & 2,44 & 3,67 & 1,01 & 68 \\
\hline \multirow[t]{2}{*}{37} & V. Ramos & Universidad Islas & 9 & 2,44 & 4,00 & 0,92 & 70 \\
\hline & & Baleares & & & & & \\
\hline \multirow[t]{2}{*}{38} & J.A. Jiménez & Univ. Politécnica de & 9 & 2,78 & 4,89 & 0,66 & 76 \\
\hline & & Catalunya & & & & & \\
\hline 39 & J.I. Castillo-Manzano & Universidad de Sevilla & 8 & 1,00 & 2,00 & 4,00 & 9 \\
\hline \multirow[t]{2}{*}{40} & H. San Martín & Universidad de & 8 & 1,50 & 2,25 & 2,37 & 24 \\
\hline & & Cantabria & & & & & \\
\hline \multirow[t]{2}{*}{41} & B. Ortega Aguaza & Universidad de & 8 & 1,63 & 2,25 & 1,89 & 37 \\
\hline & & Málaga & & & & & \\
\hline \multirow[t]{2}{*}{42} & J.B. Garau-Vadell & Universidad Islas & 8 & 1,88 & 2,25 & 2,18 & 29 \\
\hline & & Baleares & & & & & \\
\hline \multirow[t]{2}{*}{43} & F. Orfila-Sintes & Universidad Islas & 8 & 1,88 & 2,34 & 1,82 & 39 \\
\hline & & Baleares & & & & & \\
\hline \multirow[t]{2}{*}{44} & D.R. Medina-Muñoz & Univ. Las Palmas de & 8 & 1,25 & 2,50 & 2,56 & 21 \\
\hline & & Gran Canaria & & & & & \\
\hline \multirow[t]{2}{*}{45} & R.D. Medina-Muñoz & Univ. Las Palmas de & 8 & 1,88 & 2,50 & 1,70 & 41 \\
\hline & & Gran Canaria & & & & & \\
\hline \multirow[t]{2}{*}{46} & L. Andreu & Universidad de & 8 & 2,14 & 3,43 & 1,09 & 65 \\
\hline & & Valencia & & & & & \\
\hline \multirow[t]{2}{*}{47} & R. Rigal-I-Torrel & Universidad & 8 & 1,63 & 3,88 & 1,26 & 57 \\
\hline & & de Girona & & & & & \\
\hline \multirow[t]{2}{*}{48} & M.F. Schmitz & Univ. Complutense de & 8 & 3,00 & 4,63 & 0,58 & 77 \\
\hline & & Madrid & & & & & \\
\hline
\end{tabular}


Tabla 1. Ranking de autores en la investigación turística española (2002-2013).

\begin{tabular}{|c|c|c|c|c|c|c|c|}
\hline Orden general & Autores & Institución & $\begin{array}{l}\text { Trabajos } \\
\text { Totales (n) }\end{array}$ & $\begin{array}{l}\text { Primera } \\
\text { autoría }\end{array}$ & Co-autoría & Ratio líder & Orden líder \\
\hline 49 & R. Huete & $\begin{array}{l}\text { Universidad de } \\
\text { Alicante }\end{array}$ & 7 & 1,43 & 1,86 & 2,63 & 19 \\
\hline 50 & E. Ruiz-Ballesteros & $\begin{array}{c}\text { Universidad } \\
\text { Pablo de Olavide }\end{array}$ & 7 & 1,14 & 2,14 & 2,87 & 15 \\
\hline 51 & M. Sánchez-Rivero & $\begin{array}{l}\text { Universidad de } \\
\text { Extremadura }\end{array}$ & 7 & 1,71 & 2,14 & 1,91 & 36 \\
\hline 52 & F. Vera-Rebollo & $\begin{array}{l}\text { Universidad de } \\
\text { Alicante }\end{array}$ & 7 & 1,71 & 2,43 & 1,68 & 42 \\
\hline 53 & L. Pou Garcías & $\begin{array}{c}\text { Universidad Islas } \\
\text { Baleares }\end{array}$ & 7 & 2,14 & 2,57 & 1,27 & 53 \\
\hline 54 & $\begin{array}{l}\text { M. Santana- } \\
\text { Gallego }\end{array}$ & $\begin{array}{c}\text { Universidad Islas } \\
\text { Baleares }\end{array}$ & 7 & 1,43 & 2,71 & 1,81 & 40 \\
\hline 55 & J.A. Campos-Soria & $\begin{array}{c}\text { Universidad de } \\
\text { Málaga }\end{array}$ & 7 & 1,71 & 2,86 & 1,43 & 47 \\
\hline 56 & A. Esteban-Talaya & $\begin{array}{l}\text { Univ. de Castilla- } \\
\text { La Mancha }\end{array}$ & 7 & 2,29 & 2,86 & 1,07 & 66 \\
\hline 57 & J. Uche-Marcuello & $\begin{array}{c}\text { Universidad de } \\
\text { Zaragoza }\end{array}$ & 7 & 1,86 & 3,00 & 1,25 & 58 \\
\hline 58 & V. Martínez-Tur & $\begin{array}{l}\text { Universidad de } \\
\text { Valencia }\end{array}$ & 7 & 1,86 & 4,14 & 0,91 & 71 \\
\hline 59 & G. Anfuso & $\begin{array}{l}\text { Universidad de } \\
\text { Cádiz }\end{array}$ & 7 & 1,86 & 4,43 & 0,85 & 74 \\
\hline 60 & F. Díaz Pineda & $\begin{array}{l}\text { Univ. } \\
\text { Complutense de } \\
\text { Madrid }\end{array}$ & 7 & 4,57 & 6,43 & 0,24 & 79 \\
\hline 61 & M. Seric & $\begin{array}{l}\text { Universidad de } \\
\text { Valencia }\end{array}$ & 6 & 1,33 & 2,33 & 1,94 & 34 \\
\hline 62 & F. Martinez-Roget & $\begin{array}{l}\text { Univ. de } \\
\text { Santiago de } \\
\text { Compostela }\end{array}$ & 6 & 1,67 & 2,50 & 1,44 & 45 \\
\hline 62 & $\begin{array}{c}\text { X.A. Rodríguez } \\
\text { González }\end{array}$ & $\begin{array}{l}\text { Univ. de } \\
\text { Santiago de } \\
\text { Compostela }\end{array}$ & 6 & 1,67 & 2,50 & 1,44 & 45 \\
\hline 64 & J.M. Hernández & $\begin{array}{l}\text { Univ. Las Palmas } \\
\text { de Gran Canaria }\end{array}$ & 6 & 1,83 & 2,50 & 1,31 & 51 \\
\hline 65 & M. Cladera & $\begin{array}{c}\text { Universidad Islas } \\
\text { Baleares }\end{array}$ & 6 & 2,00 & 2,50 & 1,20 & 63 \\
\hline 66 & $\begin{array}{l}\text { S. Sánchez- } \\
\text { Cañizares }\end{array}$ & $\begin{array}{l}\text { Universidad de } \\
\text { Córdoba }\end{array}$ & 6 & 1,83 & 2,67 & 1,23 & 62 \\
\hline 67 & $\begin{array}{l}\text { J.M. Rodríguez } \\
\text { Antón }\end{array}$ & $\begin{array}{c}\text { Univ. Autónoma } \\
\text { de Madrid }\end{array}$ & 6 & 1,67 & 2,83 & 1,27 & 54 \\
\hline 67 & $\begin{array}{l}\text { M. Gómez } \\
\text { Rodríguez }\end{array}$ & $\begin{array}{l}\text { Univ. de Castilla- } \\
\text { La Mancha }\end{array}$ & 6 & 1,67 & 2,83 & 1,27 & 54 \\
\hline 67 & J.E. Araña Padilla & $\begin{array}{l}\text { Univ. Las Palmas } \\
\text { de Gran Canaria }\end{array}$ & 6 & 1,67 & 2,83 & 1,27 & 54 \\
\hline 70 & $\begin{array}{l}\text { J. Cuñado } \\
\text { Eizaguirre }\end{array}$ & $\begin{array}{c}\text { Universidad de } \\
\text { Navarra }\end{array}$ & 6 & 1,50 & 3,00 & 1,33 & 50 \\
\hline
\end{tabular}


Tabla 1. Ranking de autores en la investigación turística española (2002-2013).

\begin{tabular}{|c|c|c|c|c|c|c|c|}
\hline Orden general & Autores & Institución & $\begin{array}{c}\text { Trabajos } \\
\text { Totales (n) }\end{array}$ & $\begin{array}{c}\text { Primera } \\
\text { autoría }\end{array}$ & Co-autoría & Ratio líder & Orden líder \\
\hline 71 & J. Lozano & $\begin{array}{c}\text { Universidad Islas } \\
\text { Baleares }\end{array}$ & 6 & 2,00 & 3,00 & 1,00 & 69 \\
\hline 72 & B. Moliner-Velázquez & $\begin{array}{l}\text { Universidad de } \\
\text { Valencia }\end{array}$ & 6 & 2,33 & 3,00 & 0,86 & 73 \\
\hline 73 & F. Pérez de Gracia & $\begin{array}{l}\text { Universidad de } \\
\text { Navarra }\end{array}$ & 6 & 2,83 & 3,00 & 0,71 & 75 \\
\hline 74 & A. García-Pozo & $\begin{array}{l}\text { Universidad de } \\
\text { Málaga }\end{array}$ & 6 & 1,33 & 3,17 & 1,42 & 48 \\
\hline 75 & J.L. Sánchez-Ollero & $\begin{array}{l}\text { Universidad de } \\
\text { Málaga }\end{array}$ & 6 & 1,83 & 3,17 & 1,03 & 67 \\
\hline 76 & M.D. López-Gamero & $\begin{array}{l}\text { Universidad de } \\
\text { Alicante }\end{array}$ & 6 & 1,33 & 3,33 & 1,35 & 49 \\
\hline 77 & A. Fernández-Cortés & $\begin{array}{l}\text { Universidad de } \\
\text { Almería-CSIC }\end{array}$ & 6 & 1,33 & 3,67 & 1,23 & 60 \\
\hline 77 & J.M. Calaforra & $\begin{array}{l}\text { Universidad de } \\
\text { Almería }\end{array}$ & 6 & 1,33 & 3,67 & 1,23 & 60 \\
\hline 79 & J. Ramos & $\begin{array}{l}\text { Universidad de } \\
\text { Valencia }\end{array}$ & 6 & 3,83 & 4,17 & 0,38 & 78 \\
\hline
\end{tabular}

Fuente: Elaborado por los autores (2017).

ratio de autor líder. Todos los autores se han ordenado inicialmente por el número de trabajos publicados. Cuando dos o más autores tengan un mismo número de trabajos publicados, se ordenan por el menor ratio de co-autoría y, en el caso de seguir con las mismas cifras, se podría desempatar teniendo en cuenta el menor ratio de primera autoría entre los autores implicados. Por lo tanto, se ha establecido un doble ranking de autores prolíficos, recogido en la columna izquierda titulada orden general, y de autores prolíficos líderes en la columna de la derecha titulada orden líder.

Se puede observar que el ratio de autor líder ha cambiado en gran medida el orden de autores inicialmente expuesto a través del número de trabajos publicados. Merece resaltar la mejora de posiciones que han experimentado varios autores que se han situado entre los diez primeros del ranking, como son los casos de J. Alegre, que ha pasado del octavo al segundo puesto; B. Plaza, pasando del puesto 25 al 6; J.I. Castillo-Manzano, pasando del puesto 39 al 9; y J.M. López-Bonilla, pasando del puesto 27 al 10. Otros autores que han mejorado sus posiciones, colocándose entre los 20 primeros del ranking, son E. Ruiz-Ballesteros, pasando del puesto 50 al 15; T.E. Espino-Rodríguez, pasando del puesto 29 al 17; y R. Huete, pasando del puesto 49 al 19. Sin embargo, J.L. Nicolau se mantiene como autor líder e, incluso, afianza su posición de liderazgo considerando los ratios de primera autoría y de co-autoría. Las diferencias de este autor con el segundo clasificado en el ranking de liderazgo son mucho mayores.

Por una parte, los autores con un reducido indicador de primera autoría son relativamente pocos. Hay una veintena de autores que poseen un ratio de primera autoría por debajo de 1,5. Entre estos autores cabe destacar a A.I. Polo-Peña (1,00), B. Plaza (1,00), J.I. Castillo-Manzano (1,00), J.F. Molina-Azorín (1,05), J. Alegre (1,11), J.M. López-Bonilla (1,11), E. Ruiz-Ballesteros (1,14) y J.L. Nicolau $(1,15)$.

En cuanto a la cifra de co-autoría de los trabajos publicados por los autores prolíficos, se ha obtenido una media de 2,86 autores por artículo, siendo los principales autores que publican con un número de colaboraciones 
relativamente bajo los siguientes: J.L. Nicolau (1,52), R. Huete (1,86), B. Plaza (1,89), J.I. Castillo-Manzano (2,00), A. Mantecón (2,00), J.M. López-Bonilla (2,11), L.M. López-Bonilla (2,11), T.F. Espino-Rodríguez (2,13), E. Ruiz-Ballesteros $(2,14)$ y M. Sánchez-Rivero $(2,14)$. Todos estos autores citados están situados entre los 40 primeros autores líderes.

En la Figura 1 se presenta un mapa de posiciones de los 30 primeros autores líderes en función de los ratios de primera autoría y de co-autoría. Se ha establecido el eje de coordenadas de cada autor a partir de los valores medios de los 30 autores en cada uno de estos dos indicadores, distinguiéndose gráficamente cuatro cuadrantes. Así, por ejemplo, se puede observar que en la parte alta y a la derecha (cuadrante C2) se sitúan los diez autores líderes que superan los valores medios de primera autoría y de co-autoría respecto al resto de autores. En cambio, en el extremo opuesto (cuadrante (3), se encuentran posicionados los siete autores líderes, entre los 30 primeros del ranking, que publican menos artículos como primeras firmas y lo hacen en colaboración de más autores.

Por otro lado, una cuestión relevante en el presente análisis es el género de las autorías. Las mujeres presentan unos ratios medios ponderados de primera autoría y de co-autoría del 1,83 y del 2,73, respectivamente, frente a los ratios que ofrecen los hombres de 1,85 y 2,91. En lo que respecta al género de los autores prolíficos, se encuentran 22 mujeres y 57 hombres, con lo cual hay una representación femenina del 27,8\% del total. Entre los diez primeros investigadores del ranking de autores prolíicos se hallan cuatro mujeres y, si se aumenta hasta los veinte primeros autores, se encuentran siete mujeres. Si se observa el ranking de autores líderes, la representación femenina pasa a ser de tres mujeres entre los diez primeros autores y siguen siendo siete mujeres entre los veinte primeros autores.

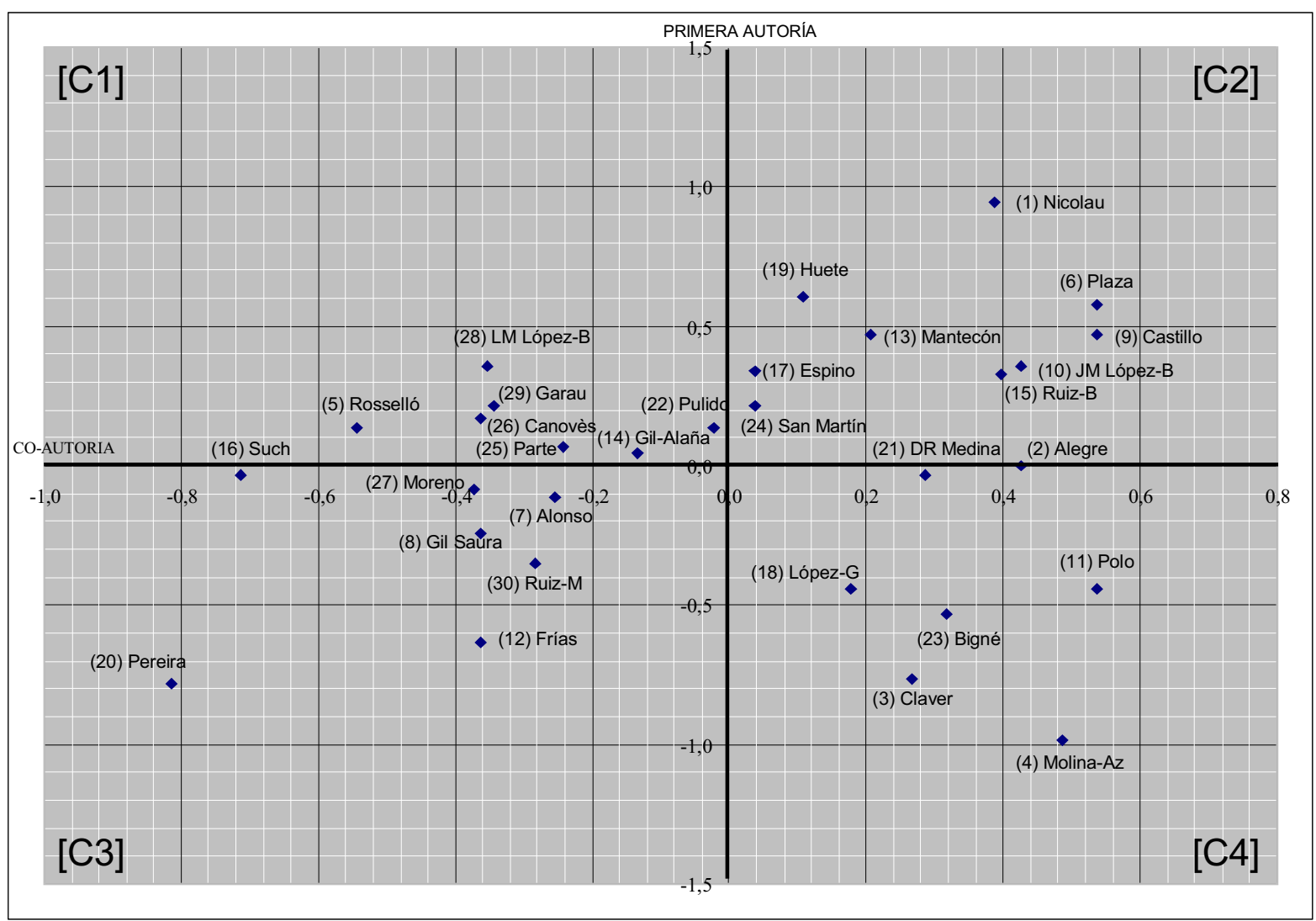

Figura 1. Representación gráfica de los 30 autores líderes principales.

Fuente: Elaborado por los autores (2017). 


\section{Discusión}

Los estudios bibliométricos sobre autoría en la investigación turística son todavía muy escasos y se han tratado de una manera tangencial. En el presente trabajo se ha realizado un análisis de autoría a través de una búsqueda muy exhaustiva y laboriosa de trabajos publicados en el ámbito turístico internacional. Con ello, se ha conseguido revisar prácticamente la totalidad de las publicaciones de autores afiliados a instituciones españolas. Salvo escasas excepciones (Corral; Cànoves, 2013; López-Bonilla; Granados-Perea; López-Bonilla, 2017; Zhong; Wu; Morrison, 2015), la gran mayoría de análisis de autoría se ha basado únicamente en revistas especializadas en turismo y, además, han utilizado un número reducido de revistas científicas, como ya han criticado algunos autores (Losekoot; Verginas; Wood, 2001). En general, los estudios bibliométricos suelen consultar menos de diez revistas científicas, siendo muy pocos trabajos los que superan esta cifra (Albacete; Fuentes; Haro-Domínguez, 2013; Mckercher, 2007; Ryan, 2005). Asimismo, los análisis bibliométricos sobre la investigación turística española se han concentrado en revistas exclusivamente españolas o se han centrado en un área de investigación muy específica (Albacete; Fuentes; Haro-Domínguez, 2013; Bigné et al., 2008; Corral; Cánoves, 2013; Hernández; Campón; Folgado, 2011; López-Bonilla; López-Bonilla, 2008; Sánchez; Marín, 2003). Por todo ello, el presente trabajo ha intentado superar todas estas limitaciones de los estudios previos aportando un amplio y profundo análisis de autorías sobre la investigación turística española.

En este sentido, este trabajo se ha basado en todas las publicaciones en revistas de prestigio, ya sean o no especializadas en turismo, con lo cual se han seleccionado y revisado 1864 artículos y se han identificado a 79 autores prolíicos en la investigación turística. Así, por ejemplo, en un estudio bibliométrico similar y muy interesante, Albacete, Fuentes y Haro-Domínguez (2013) analizan la investigación turística española de impacto internacional a través de 26 revistas especializadas en turismo, ocio y gestión de la hospitalidad durante un período de 15 años (entre 1997 y 2011), y consiguen identificar tan solo a 18 autores prolíficos con seis o más trabajos publicados en el contexto de la economía y la empresa. Por ello, es imprescindible contar con este requisito de exhaustividad en los estudios bibliométricos de esta índole para asegurar su mayor rigurosidad. Hoy en día, las bases de datos bibliográficas permiten mayores facilidades para cumplir con estas condiciones.

El presente estudio se ha enfocado hacia el análisis de autoría con el objetivo claro de establecer una distinción de los autores o investigadores líderes respecto al conjunto de autores prolíficos en la investigación turística española. Para ello, se ha realizado un análisis basado en el criterio de la primera autoría, es decir, contando el orden de las firmas de los autores en cada publicación. De este modo, se han considerado como autores líderes aquellos que firman sus publicaciones en primer orden en su mayoría de trabajos. Los autores que poseen un buen indicador de primera autoría son relativamente pocos. Por ejemplo, se hallan una veintena de autores con un ratio inferior a 1,5 en primera autoría.

En primer término, cabe destacar a J.L. Nicolau como autor prolífico líder de la investigación turística con difusión internacional. Este autor destaca por su mayor número de publicaciones, así como especialmente por su mayor esfuerzo investigador al publicar con un menor número de colaboradores en cada uno de sus trabajos, siendo primera firma en la gran mayoría de ellos. Por lo tanto, este autor demuestra un alto nivel de productividad asociado convenientemente a su liderazgo. En las ciencias sociales no es tan necesario como en las ciencias experimentales que firmen varios autores en la publicación de un artículo. No obstante, es razonable que un investigador en ciencias sociales pueda publicar algunos de sus artículos con varios colaboradores, sobre todo, cuando se realizan proyectos de investigación. Pero es menos justificable que un autor aparezca en la gran mayoría de sus artículos con numerosos firmantes. En gran parte de estos casos es posible que se trate de colaboraciones o pactos para aumentar artificialmente la producción científica. Por todo ello, sería conveniente que las valoraciones bibliométricas sobre la investigación académica, tanto personales como institucionales, tengan en cuenta 
especialmente la producción científica basada en los trabajos fraccionados, ya que demuestra, en principio, una mayor productividad de los investigadores.

En el presente estudio tan solo aparecen diez autores prolíficos que publican con un número similar de firmas que la media obtenida por Zhao y Ritchie (2007), que fue de 2,1 autores por trabajo publicado. Únicamente hay diez autores prolíficos que publiquen con una cifra similar de colaboradores, lo que supone tan solo un 11,4\% sobre el total de autores del ranking. Si se observan las posiciones medias que ocupan el conjunto de estos diez autores, llama la atención que se sitúe en el puesto 32 en el ranking general, en función del número total de publicaciones, y se coloque en el puesto 15, conforme al ranking de liderazgo. Esto puede indicar que los autores líderes contribuyen a reducir el número de co-autoría debido a su mayor capacidad y autonomía para llevar a cabo sus propios trabajos de investigación. En este sentido, es necesario que disminuya el número de autores por cada trabajo publicado, acercándose más a la media internacional, es decir, a dos autores por artículo. Los organismos españoles de evaluación de la investigación deben considerar también el hecho de la co-autoría para valorar de manera más precisa los trabajos publicados en ciencias sociales, incentivando así a aquellos autores que realizan un mayor esfuerzo investigador en sintonía con el panorama internacional.

Se ha comprobado también la existencia de desigualdad de género en la investigación turística. La representación femenina es relativamente baja todavía, alcanzando a un 27,8\% del total de autores prolíficos. Esto puede ser debido a la incorporación más tardía de la mujer respecto a las publicaciones científicas en el ámbito turístico, pero también cabe pensar en la posibilidad de un menor desarrollo de la conciliación laboral en España, así como la permanencia de un desequilibrio de género en cuanto a las responsabilidades familiares. Sin embargo, hay que considerar positivamente que la cuota relativa de mujeres entre los veinte primeros investigadores en turismo (13,6\% del total de autoras), tanto en el ranking de autores prolíficos como en el ranking de autores líderes, supera ligeramente a la cuota relativa de hombres (12,3\% del total de autores masculinos). Asimismo, las mujeres presentan unos ratios medios de primera autoría y co-autoría algo mejores que los hombres. Por lo tanto, las autoras suelen publicar más frecuentemente como primeras firmantes y, sobre todo, con un número menor de colaboradores que los hombres.

\section{Conclusión}

La difusión internacional de la investigación turística española presenta un desarrollo muy reciente. En general, el número de publicaciones internacionales mantiene una tendencia de crecimiento continuo, especialmente marcada por la entrada en vigor de la Ley de Universidades a finales de 2001, que ha actuado como la verdadera impulsora de esta evolución al exigir publicaciones internacionales a los profesores e investigadores para alcanzar el reconocimiento de su carrera docente e investigadora. La gran mayoría de autores han publicado sus trabajos en revistas de prestigio internacional a partir del segundo período analizado (2008-2013). De hecho, la media de años de antigüedad de las publicaciones internacionales de todos los autores prolíficos citados es de 4,5 años.

Los estudios bibliométricos sobre la investigación turística española e internacional se han limitado normalmente a seleccionar tan solo una serie de revistas especializadas en turismo. Esto se justifica por razones operativas a la hora de facilitar la búsqueda bibliográfica. Sin embargo, esta estrategia conlleva también la ausencia de trabajos publicados en otras revistas científicas de prestigio no especializadas en turismo, pero que pueden tener un impacto y una representatividad relevantes en la investigación turística. Por ello, el presente trabajo se ha basado en todas las publicaciones en revistas de prestigio, seleccionando y revisando un total de 1864 artículos e identificando a 79 autores prolíficos en la investigación turística. Asimismo, se ha llevado a cabo una metodología basada especialmente en el orden de firmas de los investigadores para establecer un ranking de autores líderes en la investigación turística. Se ha comprobado que el ranking de autores líderes difiere en gran medida del ranking de autores prolíficos. 


\section{Colaboraciónes}

Todos los autores contribuyeron en la concepción y desarrollo del estudio, análisis de datos y redacción final.

\section{Referencias}

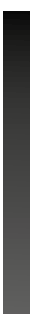

Albacete, C. A.; Fuentes, M. M.; Haro-Domínguez, M. C. La investigación española en turismo con impacto internacional (1997-2011). Una perspectiva de la economía y la dirección de la empresa. Cuadernos de Economía y Dirección de la Empresa, v. 16, n. 1, p. 17-28, 2013.

Bigné, J. E. et al. A. Investigación internacional en marketing turístico: análisis de contenido sobre temas y metodologias. PASOS. Revista de Turismo y Patrimonio Cultural, v. 6, n. 3, p. 391-398, 2008.

Brown, C. L.; Chan, K. C.; Lai, P. Marketing journal coauthorships: An empirical analysis of coauthor behavior. Journal of Marketing Education, v. 28, n. 1, p. 17-25, 2006.

Corral, J. A.; Cànoves, G. La investigación turística publicada en revistas turísticas y no turísticas: análisis bibliométrico de la producción de las universidades catalanas. Cuadernos de Turismo, n. 31, p. 55-81, 2013.

Díaz-Campos, J. Análisis bibliométrico de las tesis doctorales sobre Internet en las universidades españolas (1996-2011). Transinformação, v. 28, n. 3, p. 33-348, 2016. http://dx.doi. org/10.1590/2318-08892016000300008

He, B.; Ding, Y.; Yan, E. Mining patterns of author orders in scientific publications. Journal of Informetrics, v. 6, n. 3, p. 359-367, 2012.

Hernández, J. M.; Campón, A. M.; Folgado, J. A. La investigación en turismo en España através de las revistas de dirección de empresas. Revista de Análisis Turístico, n. 12, p. 1-9, 2011.

López-Bonilla, J. M.; López-Bonilla, L. M. Producción científica española en marketing turístico. Estudios Turísticos, n. 177 , p. 35-50, 2008.

López-Bonilla, J. M.; Granados-Perea, C.; López-Bonilla, L. M. Primera generación de autores con difusión internacional en la investigación turística española. Revista Española de Documentación Científica, v. 40, n. 3, e178, 2017.

Losekoot, E.; Verginas, C. S.; Wood, C. Out for the count: Some methodological questions in publications counting literature.
International Journal of Hospitality Management, v. 20, n. 3 p. 233-244, 2001.

McKercher, B. An analysis of prolific authors. Journal of Hospitality and Tourism Education, v. 19, n. 2, p. 23-30, 2007.

McKercher, B. A citation analysis of tourism scholars. Tourism Management, v. 29, n. 6, p. 1226-1232, 2008.

Organización Mundial del Turismo. Panorama OMT del turismo internacional. Edición 2016. [S.I]: UNWTO, 2016. Disponible en: <http://www.e-unwto.org/doi/pdf/10.18111/97892844 18152>. Acceso en: 24 jan. 2017.

Park, K. et al. Hospitality and tourism research rankings by authors, university, and country using six major journals: The first decade of the new millennium. Journal of Hospitality and Tourism Research, v. 35, n. 3, p. 381-416, 2011.

Ruiz-Pérez, R.; Marcos-Cartagena, D.; López-Cózar, E. D. La autoría científica en las áreas de ciencia y tecnología. Políticas internacionales y prácticas editoriales en las revistas científicas españolas. Revista Española de Documentación Científica, v. 37, n. 2, p. 1-11, 2014.

Ryan, $C$. The ranking and rating of academics and journals in tourism research. Tourism Management, v. 26, n. 5, p. 657-662, 2005.

Sánchez Pérez, M.; Marín Carrillo, M. B. La investigación en turismo y economía de la empresa publicada en revistas especializadas españolas: 1996-2001. Papers de Turisme, n. 33, p. 6-39, 2003.

Zhao, W.; Ritchie, J. R. B. An investigation of academic leadership in tourism research: 1985-2004. Tourism Management, v. 28, n. 2, p. 476-490, 2007.

Zhong, L.; Wu, B.; Morrison, A. M. Research on China's tourism: A 35-year review and authorship analysis. International Journal of Tourism Research, v. 17, n. 1, p. 25-34, 2015. 